\title{
Owe Period Fast North-Seeking Method Based On Interpolation Of Polynomial
}

\author{
Li Zhang, Lilong Tan, Qiyuan Zhong \\ The Rocket Force University of Engineering, Xi'an 710025,China
}

Keywords: polynomial interpolation; owe period; sinusoid ; gyroscope

\begin{abstract}
In order to make rationally use of the original data and improve the data fitting precision of the sine waveform, the owe period fast North-Seeking method of the polynomial interpolation is based on the least square method. According to the character of the large deflection north point data, the polynomial interpolation subdivision is carried out based the eighth data fitting a quarter data. To owe period fitting of the quarter data of sinusoidal parameter. Use a certain type gyro theodolite as prototype.Through software simulation algorithm ,the experimental results show that the algorithm reasonable use of the data,shortened the time for the north,improves the fitting accuracy.
\end{abstract}

\section{INTRODUCTION}

The north seeking technology has been widely used in the fields of aeronautics and astronautics, mining, submarine tunnel,geographic mapping, energy exploration and military affairs ${ }^{[1]}$.At present,the development direction of gyro theodolite is high precision, fast and full automation,but high precision and fast is often a pair of contradictory requirements. One of the methods to solve this problem is to divide the files.

It is difficult for the whole tribe to detect the sensitivity of the whole tribe in the detection area of the photoelectric detection element, which is influenced by the North position, the locking mechanism and the decentralization stability.In order to ensure the accuracy of north seeking, it is usually required to swing the whole tribe in the range of the photoelectric sensitive area, which is convenient for monitoring, calculation and data processing ${ }^{[2]}$.Therefore, proper methods must be used to limit the amplitude of the pendulum. At present, the electromagnetic damping method and step method are usually used to limit the gyro sensitivity ${ }^{[3]}$.

Bai Yunchao, et al. In the paper "the design of the damping torque in the automatic gyro theodolite" (Journal of Xi'an University of Science And Technology, 2009.11, 29) (:752-755.), it is pointed out that there are more than three problems in the electromagnetic damping method:

1. The dynamic coil is fixed on the gyro sensitive part, so that the structural dimension of the gyro sensitive part is increased in the length direction, which leads to the increase of the overall size of the instrument, and has certain influence on the symmetry of the gyro sensitive part structure;

2. Because the gyro sensitive part must be put into the follow-up cylinder, the stator coil can be fixed on the cylinder wall of the servo cylinder, and the installation of the stator coil is very inconvenient;

3. It is necessary to increase the power of the two guide wires for the rotor coil, too much of the guide wire will affect the stability of the gyro sensitivity.

Wang Zhen, etal. In the literature "integral measurement method of pendulous gyroscope" (Optics and precision engineering, 2007.05, 15 (5):746-752) is described in detail in the step principle.The step method has the advantages of good stability and no additional requirements for the hardware, but the limiting time of the step method is too long, which limits the further shortening of the overall search time. Therefore, how to realize the fast limit is one of the key problems of the gyro north seeking technology.

\section{Measuring Principle of Gyro North Finder}

Pendulous gyroscope using metal sling hanging a center of gravity in the gyro sensitive parts, in the comprehensive effect of heavy torque and rotation of the earth, around the north near the gyro spindle reciprocating swing, according to the law of motion of the gyroscope axis. Through the 
equilibrium position detection of gyroscope rotation, you can determine the direction of true north [4-6] Gyro spindle motion state detection principle as shown in Figure 1: The gyro sensitive part 5 is internally equipped with a gyroscope rotor 6, through the outside of the guide wire 3 to the gyroscope rotor 6 power supply, so that the gyroscope rotor rotor rotation along the rotor axis and angular momentum $\mathrm{H}$, the direction of the angular momentum $\mathrm{H}$ is the direction of the gyro spindle; The suspension column 4 and 5 gyro sensitive parts is fixedly connected by 1 sling hanging in the north finder housing 10; mirror 2 optical adhesive in the suspension column 4 upper and 5 gyro sensitive parts can rotate around the sling 1;The light emitted by the laser 9 is received by the photoelectric detecting element 8 after being reflected by the swing mirror 2, and the photoelectric detecting element 8 converts the optical signal into an electrical signal, and the corresponding signal is processed by the processor 7; When the gyro sensitive parts 5 around 1 sling rotates, the light from the mirror 2 reflection formed after the cursor will like the photoelectric detection element 8 to produce the corresponding movement, the position corresponding to the cursor like gyroscope axis; The rotation of the instrument housing is controlled by the rotation of the rotary motor 11 after the instruction given by the processor 7 [7]。

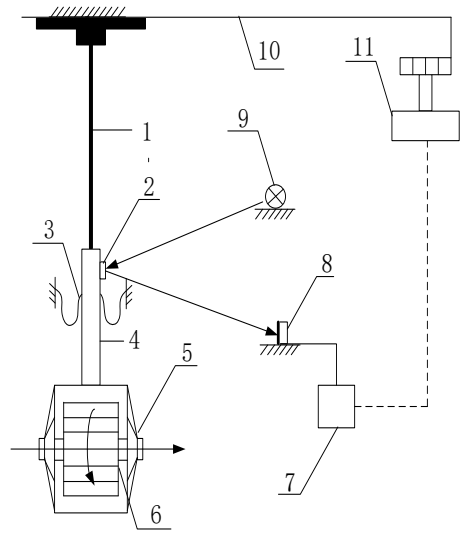

FIGURE 1. pendulous gyroscope north finder measurement principle structure

\section{Improved Clipping Scheme}

Rough north seeking after the decentralization of gyro sensitive parts, when the gyro sensitive to precession, by rotating the sling hanging point to drive the torsion torque generated by the sling, sling to change the gyro swing track, up to three times by rotating the sling hanging point to swing, with zero point reverse suspension and coarse north basic coincide effect of the gyro sensitive parts in north direction near the little swing [8-10].

This scheme by detecting the non complete cycle of gyroscopic pendulum cursor movement, fitting complete gyroscope swing equation and determine the direction to establish a fast coarse north; limiting model, get the instrument shell three rotational moment, direction and angle; Through the precise control of rotary motor, the swing reversal point, zero and one sling coincide, in the north near the gyro swing slightly, complete limiting [11]. Specific steps are as follows:

Step one: decentralization of gyro sensitive parts 5, non full cycle gyro based on photoelectric detecting element 8 detected 5 swing position sensitive cursor signal sequence $X_{n}$ and the corresponding time series $t_{n}$, calculating the coarse north direction ${ }^{\alpha_{N}}$ and oscillation of gyro sensitive parts equation;

1. Open the instrument power supply, complete the instrument self-test, enter the local latitude value, start the gyro rotor to achieve rated speed;

2. Decentralization of gyro sensitive parts, by the acquisition of photoelectric detection element position time period of at least $1 / 4$ gyroscope gyro sensitive sequence cursor $x_{1}\left(t_{1}\right) 、 x_{2}\left(t_{2}\right)$ 、 $x_{3}\left(t_{3}\right) \ldots x_{M}\left(t_{M}\right)$, Common M groups of data; 
3: According to the position sequence acquisition ${ }^{x_{n}}\left(t_{n}\right)$, analysis of the coarse north direction $\alpha_{n}$ and oscillation of gyro sensitive parts equation $\alpha(t)$;

Step two: according to the ministry of gyro sensitive initial swing equation of gyro north finder limit control model, get the instrument shell three rotational moments $t_{0} t_{1} t_{2}$, the corresponding rotation angle $\Delta \alpha_{1} \Delta \alpha_{2} \Delta \alpha_{3}$, and instrument calibration value measuring system $A_{0} A_{1} A_{2} A_{3}$ to precisely control the rotary motor. Because each shell rotation speed than the spindle speed gyroscope precession, so it can be considered that the rotation in a moment, ignored the effect of time difference;

1. When the system calculates the time of the three rotation and the corresponding angle, the time is recorded as $t_{0}$, and the reading of the instrument angle is $A_{0}$. The first instrument shell rotates quickly The direction of rotation and the angle of rotation are determined by the following methods: as shown in Figure 3.2 and figure 4.2, the rotary motor drives the instrument shell to make gyroscope precession direction of rotating spindle North $\Delta \alpha_{1}=\alpha_{N}$. The instrument readings for measuring system is $A_{1}, A_{1}=A_{0}+\Delta \alpha_{1}$, To ensure that the instrument after turning main spindle swing gyroscope two new reversal points in the north on both sides;

(1). By the steps of type (3) the gyro sensitive relative initial photoelectric ${ }{ }_{0}(t)$ detection element zero angular displacement can be described as:

$$
x_{0}(t)=L_{0} \sin \left(\omega t+k_{0}\right)+d_{0}
$$

The initial reading of the instrument angle measuring system is $\mathrm{A} 0$, and the rotary motor drives the instrument shell to rotate to the position of $A_{1}=A_{0}+\Delta \alpha_{1}$. Because of the relative precession of gyro sensitive parts, instrument shell rotation speed is very fast, can be considered to be instantaneous, so the gyro axis due to inertia, speed is constant;

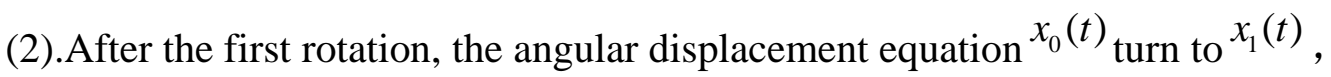

$$
x_{1}(t)=L_{1} \sin \left(\omega t+k_{1}\right)+d_{1}
$$

The rotation before and after the meet:

$$
\begin{gathered}
x_{1}\left(t_{0}\right)=x_{0}\left(t_{0}\right)+\lambda \Delta \alpha_{1} \\
x_{1}^{\prime}\left(t_{0}\right)=x_{0}^{\prime}\left(t_{0}\right) \\
d_{1}=d_{0}-\lambda \Delta \alpha_{1}
\end{gathered}
$$

By formula (12) (14) can be pushed:

$$
\begin{gathered}
k_{1}=\arctan \left(\frac{\omega\left(A_{0} \sin \left(\omega t_{0}+k_{0}\right)+\lambda \Delta \alpha_{1}\right)}{A_{0} \omega \cos \left(\omega t_{0}+k_{0}\right)}\right)-\omega t_{0} \\
L_{1}=\frac{L_{0} \cos \left(\omega t_{0}+k_{0}\right)}{\cos \left(\arctan \left(\frac{L_{0} \sin \left(\omega t_{0}+k_{0}\right)+\lambda \Delta \alpha_{1}}{L_{0} \cos \left(\omega t_{0}+k_{0}\right)}\right)\right)} \\
d_{1}=d_{0}-\lambda \Delta \alpha_{1}
\end{gathered}
$$

The type (15) (17) into the equation (11) can have a rotating angular displacement equation $x_{1}(t)$.

2. When the gyro sensitive to precession $t_{1}$ moment, rotary motor driven instrument casing to rotate by the $A_{1}$ position to the $A_{2}$ position, $A_{2}=A_{1}-\Delta \alpha_{2}$. Figure 3.3 and figure 4.3 , second times of rotation after the oscillation of gyro sensitive parts have a reversal point $C_{2}$ (or 
coincidence $^{C_{2}^{\prime}}$ ) and North $\mathrm{N}$;

(1). After the second rotation, the angular displacement equation is changed from A to $B$, After the second rotation, the angular displacement equation is changed from $x_{1}(t)$ to $x_{2}(t)$,

$$
x_{2}(t)=L_{2} \sin \left(\omega t+k_{2}\right)+d_{2}
$$

The rotation before and after the meet:

$$
\begin{gathered}
x_{2}\left(t_{1}\right)=x_{1}\left(t_{1}\right)+\lambda \Delta \alpha_{2} \\
x_{2}^{\prime}\left(t_{1}\right)=x_{1}^{\prime}\left(t_{1}\right) \\
d_{2}=d_{1}-\lambda \Delta \alpha_{2}=d_{0}-\lambda\left(\Delta \alpha_{1}+\Delta \alpha_{2}\right)
\end{gathered}
$$

By formula (19) (21) can be pushed:

$$
\begin{aligned}
k_{2} & =\arctan \left\{\frac{\left[L_{1} \sin \left(\omega t_{1}+k_{1}\right)+\lambda \Delta \alpha_{2}\right]}{L_{1} \cos \left(\omega t_{1}+k_{1}\right)}\right\}-\omega t_{1} \\
L_{2}\left(t_{1}\right) & =\frac{L_{1} \cos \left(\omega t_{1}+k_{1}\right)}{\cos \left\{\arctan \left[\frac{L_{1} \sin \left(\omega t_{1}+k_{1}\right)+\lambda \Delta \alpha_{2}}{L_{1} \cos \left(\omega t_{1}+k_{1}\right)}\right]\right\}} \\
d_{2} & =d_{1}-\lambda \Delta \alpha_{2}=d_{0}-\lambda\left(\Delta \alpha_{1}+\Delta \alpha_{2}\right)
\end{aligned}
$$

The type (22) (24) into the equation (18) obtained $x_{2}(t)$;

1). As shown in Figure 3.1, when the two reversal points of gyro sensitive parts in the same initial swing north side, in order to make second times after the rotation of gyro sensitive parts swing a reversal point coincides $C_{2}^{\prime}$ with the N, as shown in Figure 3.3, to meet the needs of:

$$
\angle B_{1} O B_{2}=\angle B_{1} O C_{2}^{\prime}+\angle C_{2}^{\prime} O B_{2}
$$

According to figure 3.3, the formula (25) is introduced:

$$
\begin{aligned}
\angle B_{1} O N+\angle N O B_{2} & =\lambda \angle O_{1} O C_{2}^{\prime}+\angle C_{2}^{\prime} O B_{2} \\
\lambda \angle O_{1} O N+\lambda \angle N O O_{2} & =\lambda\left(\angle O_{0} O O_{1}-\angle O_{0} O N\right)+\angle C_{2}^{\prime} O B_{2} \\
\lambda \angle O_{1} O O_{2}= & \lambda\left(\angle O_{0} O O_{1}-\angle O_{0} O N\right)+\angle C_{2}^{\prime} O B_{2} \\
\text { is: } \quad & -\lambda \Delta \alpha_{2}=\lambda\left(\Delta \alpha_{1}-\alpha_{N}\right)+L_{2}\left(t_{1}\right)
\end{aligned}
$$

That

(17)

2). As shown in Figure 4.1, when the gyro sensitive parts of the two initial swing reversal point is not in the same side one, in order to make second times after the rotation of gyro sensitive parts swing a reversal point $C_{2}$ and $N$ overlap, as shown in Figure 4.3, to meet the needs of:

$$
\angle B_{1} O B_{2}=\angle B_{1} O C_{2}+\angle C_{2} O B_{2}
$$

According to figure 4.3, the formula (27) is introduced:

$$
\begin{aligned}
\angle B_{1} O N+\angle N O B_{2} & =\lambda \angle O_{1} O C_{2}+\angle C_{2} O B_{2} \\
\lambda \angle O_{1} O N+\lambda \angle N O O_{2} & =\lambda\left(\angle O_{0} O O_{1}+\angle O_{0} O N\right)+\angle C_{2} O B_{2} \\
\lambda \angle O_{1} O O_{2} & =\lambda\left(\angle O_{0} O O_{1}+\angle O_{0} O N\right)+\angle C_{2} O B_{2}
\end{aligned}
$$

That is: $\lambda \Delta \alpha_{2}=\lambda\left(\alpha_{N}-\Delta \alpha_{1}\right)+L_{2}\left(t_{1}\right)$

(2). The type (23) into equation (26) or (28), $\Delta \alpha_{2}\left(t_{1}\right)$ can get uniform expression:

$$
\Delta \alpha_{2}\left(t_{1}\right)=\frac{\lambda^{2}\left(\Delta \alpha_{1}-\alpha_{N}\right)^{2}-L_{1}^{2}}{2 \lambda\left[L_{1} \sin \left(\omega t_{1}+k_{1}\right)+\lambda\left(\alpha_{N}-\Delta \alpha_{1}\right)\right]}
$$


Including: L1, K1 by type (15), (16) determined

(3). The maximum value of ${ }^{t_{1}}$ to determine the time decided by $t_{1}$, is the maximum allowed angle sling to reverse, and the structural parameters of gyroscope stop mechanism on;

(4): The rotary motor drives the rotation of the housing by the instrument ${ }^{A_{1}}$ position to the $A_{2}$ position, oscillation of gyro sensitive parts have a reversal point $C_{2}$ (or $C_{2}^{\prime}$ ) coincides with the North N.

3.Figure 3.4 or figure 4.4 , gyro sensitive to precession, $t_{2}$ moment rotary motor drives the instrument shell towards the north to coincide with the reversal point of the direction of rotation, the theodolite dial reads from $A_{2}$ to $A_{3}, A_{3}=A_{2}+\Delta \alpha_{3}$,

$$
\omega t+k_{2}=\omega\left(t-t_{1}\right)+\arctan \left\{\frac{\left[L_{1} \sin \left(\omega t_{1}+k_{1}\right)+\lambda \Delta \alpha_{2}\right]}{L_{1} \cos \left(\omega t_{1}+k_{1}\right)}\right\}
$$

$t_{2}$ is the solution to satisfy $t \geq t_{1}$ and $=\frac{\pi}{2}$ 或 $\frac{3 \pi}{2}$

The analysis obtained from figure 3.1 3.4 or 4.1 4.4: $\Delta \alpha_{3}=\Delta \alpha_{2}-\left|\alpha_{N}-\alpha_{1}\right|$;

Step3:According to step 2 to get the instrument shell three rotational moments $t_{0} t_{1} t_{2}$, the corresponding rotation angle $\Delta \alpha_{1} \Delta \alpha_{2} \Delta \alpha_{3}$, and instrument calibration value measuring system $A_{0} A_{1} A_{2} A_{3}$ to control rotary motor, the swing reversal point, zero and one sling coincide, gyro swing slightly in the true north annex, complete limiting [12]。

\section{Experimental Verification}

Relying on the 1001 factory of a certain type of prototype experiments.

Step 1: decentralization of gyro sensitive parts, acquisition of 30s gyroscope cursor motion data, using sine curve fitting method to get the coarse north direction 2 degrees north, the initial sensitive equation for gyro motion:

$Y_{0}(t)=0.0145444 \sin (0.0523599 t+0.0157080)+0.0002908$

Step 2: according to the initial motion equation, the paper establishes the model of the rotation limit of the shell of the instrument, and obtains the time and angle of the three rotation of the shell;

Step 2.1:get the coarse north direction 2 degrees after 5 seconds time scale instrument angle measuring system is $9^{\circ} 27$ ' 20 ", the rotary motor drives the instrument shell towards the direction of precession gyro axis acceleration to rotate $2^{\circ} 20^{\prime}$, the measuring system of scale value is $11^{\circ}$ 27 '40";

Step 2.1: 35 seconds time rotary motor control instrument shell towards the first rotation in the opposite direction to rotate $5^{\circ} 11$ '23 ", the measuring system of scale value is $6^{\circ} 16^{\prime} 17^{\prime}$;

Step2.3:46 seconds time rotary motor control instrument shell toward the direction of rotation of the first rotating $4^{\circ} 51$ '23 ", the measuring system of scale value is $11^{\circ} 07^{\prime} 40^{\prime \prime}$.

Step 3: complete the limit, waiting for the fine north.

The experimental results analysis: 


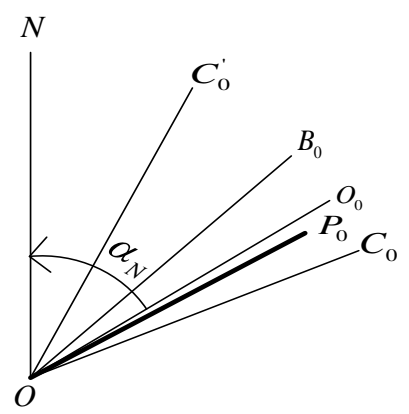

FIGURE 2.1. the initial swinging across the north gyro initial swing diagram

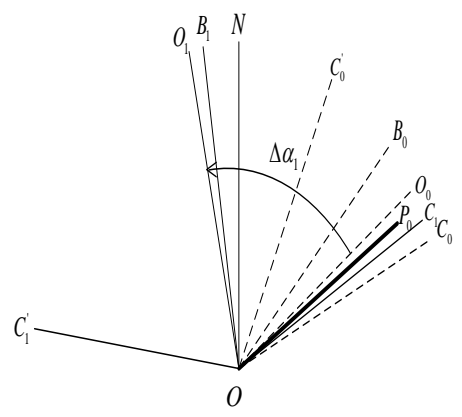

FIGURE 2.2 the first rotation of the gyro case when the initial oscillation does not cross the North

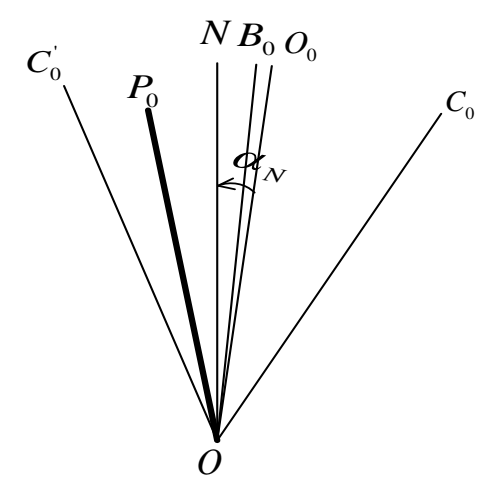

FIGURE.3.1 the initial swing of the initial swing North

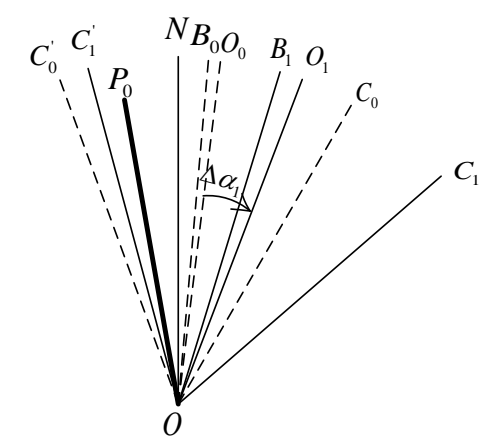

FIGURE3.2 The first rotation of the gyro shell when the initial oscillation is North 


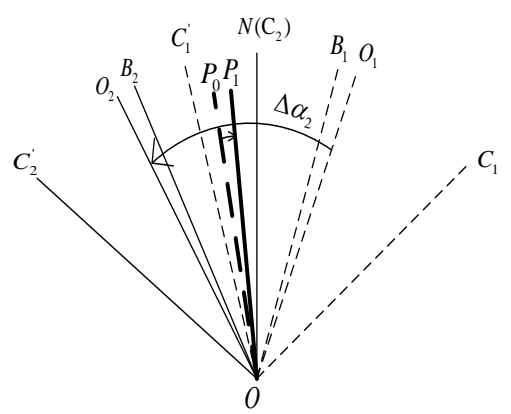

FIGURE3.3 The second rotation of the gyro shell when the initial swing is North

\section{Concluding Remarks}

At present, usually using the directional magnetic compass the initial installation direction is located in the specified range, then the coarse north seeking method corresponding to the gyro azimuth of principal axis meet the precise north seeking, finally through different precise north seeking observation method, realize high precision measurement of gyro theodolite [13].This paper focuses on the current status quo, aiming at the defects of the existing technology, provides a pendulous gyroscope fast approaching the north and limiting method, namely fast north seeking method of polynomial interpolation under cycle based on, which can make the pendulous gyroscope in half a cycle not completed within the limit, and no the additional hardware requirements, effectively solve the shortcomings of the traditional clipping method for unstable time, shorten the whole north seeking time, improve the limiting efficiency, lay a good foundation for the next work of north seeking precision.

\section{References}

[1] Zongchun Li, Guangyun Li, Guanyu Li, etal. Discussion on orientation program of GYROMAT2000 gyro theodolite [J]. Science of Surveying and Mapping, 2006, 31 (5) : 107-109.

[2] Zhingming Shen, Zeyu Zhang, Zhichao Liu. Application of GYROMAT2000 gyro theodolite [J]. Marine surveying and mapping, 2006, 26(5): 68-70.

[3] Yumin Tian, Siwei Liu, Yunchao Bai. Tilting gyro theodolite coarse search new methods [J]. Journal of Chinese Inertial Technology, 2009, 17(4): 441-448.

[4] Zhili Zhang, He Chen, Zhaofa Zhou. Composite type omnibearing fast pendulous gyro north seeking [J]. Optics and precision engineering, 2013, 21(12): 3072-3079.

[5] He Chen, Zhili Zhang, Zhaofa Zhou. Speed detection method of pendulous gyroscope full range rapid pre orientation [J]. Journal of instrument and meter, 2014, 35(10): 2378-2383.

[6] Guoying Li, Zhili Zhang, Zijie Wang. Application of compound north seeking in automatic gyro theodolite system [J]. Sensor Technology, 2005, 24(10): 77-79,85.

[7] Li Feng, Guimei Dong, Yuchi Lin, etal. Fast north seeking based on Intelligent gyro theodolite [J]. Optics and precision engineering, 2009, 17(7): 1582-1586.

[8] Li Feng, Guimei Dong, Yuchi Lin. Trend prediction 1/8 cycle fast north seeking method [J]. Nanotechnology and precision engineering, 2011, 9(1): 53-57.

[9] Guisuo Xia, Mingchun Lin, Yuchi Lin, etal. Intelligent gyro north seeking by 1/4 period integration method [J]. Journal of sensing technology, 2007, 20(8): 1926-1929.

[10]Guisuo Xia, Mingchun Lin, Yuchi Lin, etal. Study on Application of half period transit method 
in north seeking [J]. Journal of sensing technology, 2007，20(4): 781-784.

[11]Guisuo Xia, Mingchun Lin, Yuchi Lin, etal. Intelligent gyro north seeking with 3/4 period transit method [J]. Sensors and micro systems, 2007, 26(9): 53-55.

[12]Guisuo Xia, Mingchun Lin, Yuchi Lin, etal. Real time transit north seeking algorithm [J]. Nanotechnology and precision engineering, 2007, 5(4): 315-318.

[13]Guisuo Xia, Mingchun Lin, Yuchi Lin, etal. Research on the application of integral method in intelligent gyro north seeking system [J]. Sensors and micro systems, 2007, 26(10): 57-59. 